\title{
MRI Slice Segmentation and 3D Modelling of Temporomandibular Joint Measured by Microscopic Coil
}

\author{
O. Smirg ${ }^{1}$, O. Liberda ${ }^{2}$, Z. Smekal ${ }^{1}$, A.Sprlakova-Pukova ${ }^{3}$ \\ ${ }^{1}$ Department of Telecommunication, Faculty of Electrical Engineering and Communication, University of Technlogy, \\ Purkynova 118, 612 00, Brno, Czech republic \\ ${ }^{2}$ Department of Oral and Maxillofacial Surgery, Faculty of Medicine, Masaryk University and Faculty Hospital Brno, \\ Kamenice 5, 625 00, Brno, Czech republic \\ ${ }^{3}$ Department of Radio-diagnostics, Faculty of Medicine, Masaryk University and Faculty Hospital Brno, Kamenice 5, \\ 625 00, Brno, Czech republic \\ smirg@feec.vutbr.cz, oliberda@fnbrno.cz,smekal@feec.vutbr.cz, asprlakova@fnbrno.cz
}

\begin{abstract}
The paper focuses on the segmentation of magnetic resonance imaging (MRI) slices and 3D modelling of the temporomandibular joint disc in order to help physicians diagnose patients with dysfunction of the temporomandibular joint (TMJ). The TMJ is one of the most complex joints in the human body. The most common joint dysfunction is due to the disc. The disc is a soft tissue, which in principle cannot be diagnosed by the CT method. Therefore, a 3D model is made from the MRI slices, which can image soft tissues. For the segmentation of the disc in individual slices a new method is developed based on spatial distribution and anatomical TMJ structure with automatic thresholding. The thresholding is controlled by a genetic algorithm. The 3D model is realized using the marching cube method.
\end{abstract}

Keywords: Marching cubes, genetic algorithms, magnetic resonance imaging, Iso-surfaces, 3D models

\section{INTRODUCTION}

$T^{1}$ HE IMPORTANCE of post-processing imaging methods in medicine continues to grow. This can in part be attributed to modern non-invasive diagnostic methods, which mainly include the magnetic resonance (MR) method, and the computer tomography (CT) method. Each of these methods is based on a different physical principle, and has its advantages and disadvantages. The main and greatest advantage of the MR method compared with the CT method is the possibility of non-invasive diagnosis of soft tissue. The most important part of the TMJ is the disc, which is formed by soft tissue and has an approximate length of $8 \mathrm{~mm}$. The disc is the most common cause of the TMJ dysfunction, and therefore, to obtain a more accurate diagnosis the main attention is focused on maximally accurate 3D modelling of the disc. Although modern MR tomographs can measure very thin slices with relatively high resolution, the disc appears only on a small number of images of these slices, which are, moreover, of low resolution. For example, if MRI slices are configured such that, as in our case, they are in distance of $1 \mathrm{~mm}$ apart, the disc will be visible only in eight slices. Pixel resolution in the direction of the other two axes is about $1.5 \mathrm{~mm}$. The area where the disc occurs is also partly degraded by noise. The newly proposed method reduces the adverse effects by combining the knowledge of the disc anatomical arrangement and the optimization of the MR image luminance of the disc, which is controlled by the genetic algorithm.

Currently, the diagnosis of the disc damage is based on 2D MRI slices. However, the continuity and spatial arrangement of the damaged TMJ cannot be identified from these 2D slices [1-4]. The TMJ belongs to the most complex joints in the human body. The joint realizes a rotational translational motion, which the disc determines.

If the disc has a defect, which is in most of the cases the problem with TMJ, then it can block the joint. Making a diagnosis of the TMJ disc disease is quite difficult. Creating 3D models of the TMJ disc can be of great help in establishing a correct diagnosis of TMJ diseases [5-7].

\section{TMJ PROPERTIES}

To understand the characteristics of TMJ, methods have been developed that are directly derived from anatomical assumptions [24-25]. Since it is impossible to create a universal segmentation method capable of mastering all of the existing situations of detecting the properties of the image, it is necessary to focus, at the very beginning, on a specifically set MR tomograph. In cooperation with radiologists, the MR scanner is set such that it gives the sharpest contrast between the disc and the surrounding tissue.
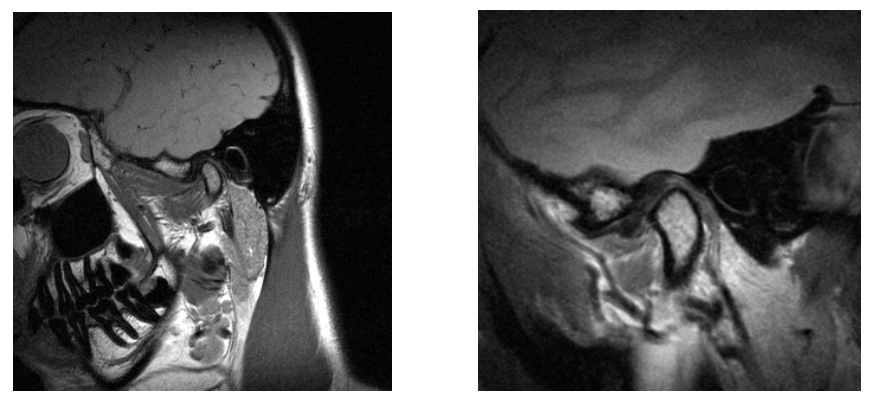

Fig.1. Examples of MRI images expressed by PD (Proton Density): left - no microscopic coil is used, right - a microscopic coil is used. 
On the basis of an expert radiologist's assessment, the algorithms for two types of images were tested. The measurements were performed using the Philips MR tomograph 9A12197 type. Data about NMR and the coils used are given in Table 1.

Table 1. Parameters of NMR

\begin{tabular}{|l|l|l|}
\hline Parameters & $\begin{array}{l}\text { Without } \\
\text { microscopic } \\
\text { coil }\end{array}$ & $\begin{array}{l}\text { With } \\
\text { microscopic } \\
\text { coil }\end{array}$ \\
\hline Magnetic Field Strength (T) & 1.5 & 1.5 \\
\hline Size of view(mm) & $150 \times 150$ & $70 \times 70$ \\
\hline Type of RF coils & C3 & micro-47 \\
\hline
\end{tabular}

First, type W-TSE PD images of the whole head were measured without the microscopic coil. Second, the same measurement was performed with the microscopic coil. Although the MR tomograph can increase the area of interest by measuring without the microscopic coil, this measurement suffers from low SNR (Signal-to-Noise Ratio). The image is completely distorted so that no adequate information about TMJ can be obtained. The examples of both MR images are shown in Fig.1 and their properties are listed in Table 2.

Table 2. Parameters of MR images

\begin{tabular}{|l|l|l|l|l|l|}
\hline $\begin{array}{l}\text { Microscopic } \\
\text { coil }\end{array}$ & $\begin{array}{l}\text { Resolution } \\
\text { (pixel) }\end{array}$ & Pixel/mm & Weighting & $\begin{array}{l}\text { Depth } \\
\text { of cut } \\
\text { (mm) }\end{array}$ & $\begin{array}{l}\text { Num- } \\
\text { ber of } \\
\text { slices }\end{array}$ \\
\hline No & $320 \times 320$ & $2.13 \times 2.13$ & $\begin{array}{l}\text { PDW- } \\
\text { TSE }\end{array}$ & 2 & 80 \\
\hline Yes & $640 \times 640$ & $9.14 \times 9.14$ & $\begin{array}{l}\text { PDW- } \\
\text { TSE }\end{array}$ & 2 & 20 \\
\hline
\end{tabular}

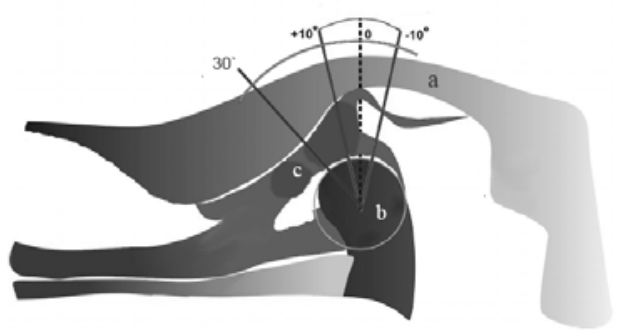

Fig.2. TMJ with the mouth closed: a) the temporomandibular socket (fossa), b) the temporomandibular condyle (head), c) the temporomandibular disc (diskus).

The most important part of the TMJ and also the most difficult to detect is the joint disc. Unfortunately, the disc is the most common case that causes problems with TMJ. Usually there is a deformation of the TMJ disc. It is because the disc is often distorted that segmentation methods based on finding image parts by their shape cannot be used. The disc, fortunately, can move only in a limited space, and therefore, the proposed method based on the likelihood of its presence can find it. As shown in Fig.2, based on medical studies [23], in healthy humans the centre of the disc is approximately 30 degrees from the top of the circle circumscribing the TMJ condyle, and the upper limit of the disc is in the range of \pm 10 degrees from the top of the circle circumscribing the TMJ condyle [6].

\section{TMJ CONDYLE SEGMENTATION}

\section{A. Segmentation of the TMJ condyle}

When searching for the disc, in the first stage we focus on finding the condyle, which, unlike the disc, has very good contrast properties. The segmentation of the condyle can be done using the marker-controlled watershed algorithm. Since the segmentation is just based on the position of the disc with respect to the head, we have to find the head first, and the segmentation of the disc can then be done only in a limited area. The result of the segmentation of the head is shown in Fig.3, [5].

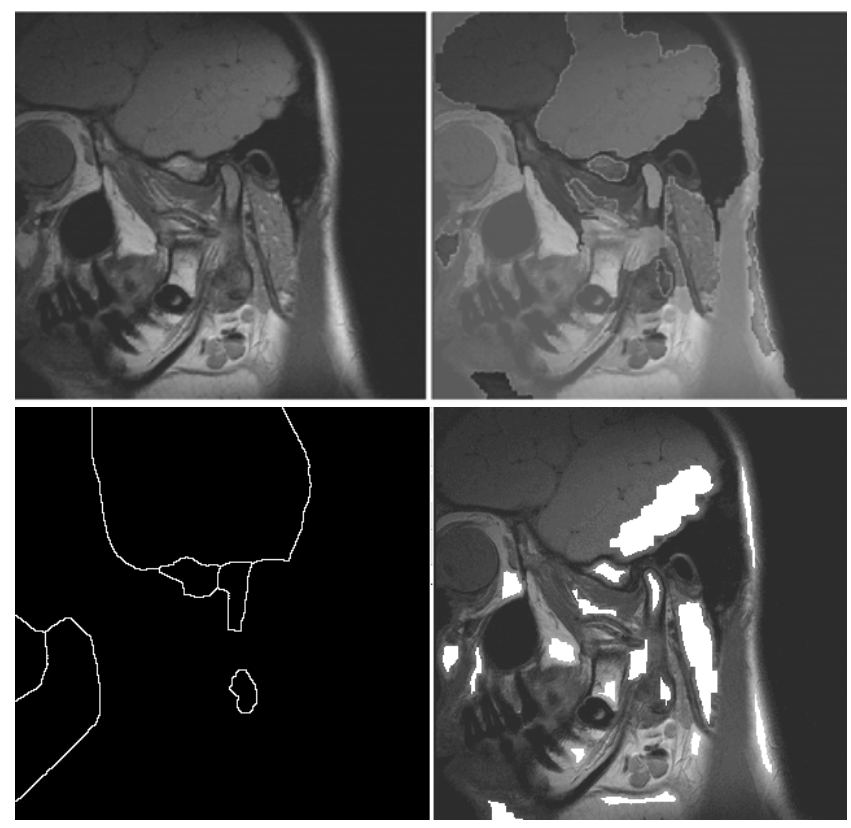

Fig.3. An example of the TMJ condyle segmentation using the marker-controlled watershed algorithm. Lower figures show defined boundaries (left) and markers (right).

The marker-controlled watershed method of segmentation is based on the idea that the image has the character of a terrain with peaks and lowlands. The segmented image parts are formed by analogy with water flooding areas. An improved method is controlled by markers, which determine the location of areas where water will flow. To determine the flooded areas a combination of methods of the type of Erosion and Dilation (Open, Close) and with edge detectors was used. To determine the boundaries the Voronoi diagram was used.

\section{B. Segmentation of the TMJ disc}

The segmentation of the disc itself is much more difficult than the segmentation of the condyle. The disc is not as contrastive against its neighbourhood as the condyle, and 
therefore the proposed method based on decreasing the area can only gradually define the space where the disc is most likely to move. In the first stage a space based on the knowledge of anatomy is created in which the disc can move even if the disc is defective. The starting point for the determination of the disc is the condyle, segmented using the marker-controlled watershed algorithm. Fig. 4 shows the limited space where the likely movement of the disc is to be found [6].

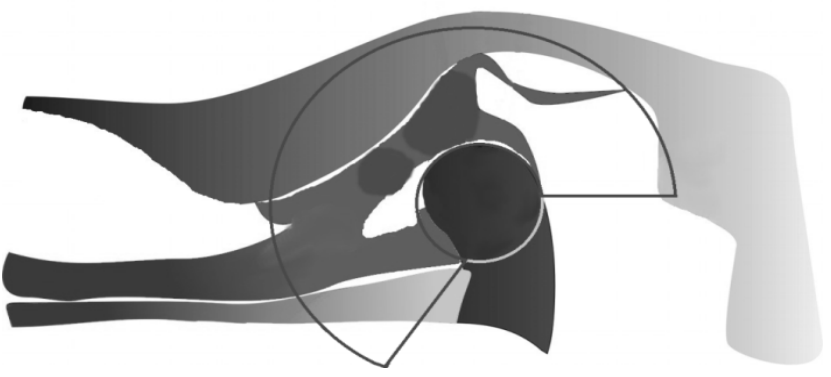

Fig.4. Definition of the space in which the disc will probably be.

The delineated region and the disc exhibit two main properties by which you can distinguish the disc from the delineated region. The first feature is the spatial character of the disc. Since only selected sections are chosen, in which the disc is included (with the first and last sections), individual layers are spatially continuous. At the same time, the surrounding tissues can also change, but they are not continuous objects, and thus they do not show spatial coherence in their range. The probability of the occurrence of space associated with the disc at the given location can be determined by the formula:

$$
p_{\text {disc }}\left(f_{k}(x, y)\right)=\sum_{k=1}^{K} \frac{f_{k}(x, y)}{K},
$$

where $f(x, y)$ is the image function in different sections, and $K$ is the number of layers. As seen from Eq.1, this is actually the average image intensity of pixels in each slice. The result is a value ranging from 0 to 1 , because it is a binary image.

An example of a probability model of spatial continuity is shown in Fig.5, where the darkest area shows the least probability of spatial continuity and the brightest area is characterized by the highest probability of spatial continuity. The disc is obviously not continuous in all sections, and therefore a threshold is chosen which determines how high the probability of the disc occurrence is. The resultant binary image is further extended by the operation of dilatation. It is one of the fundamental methods of mathematical morphology, which is based on Minkowski's addition. Due to the dilatation applied to the image the boundaries of objects in the image are spread on the basis of similarities with the current boundaries. This is done in order to avoid irreversible elimination of components related to the TMJ disc [8].



Fig.5. Definition of the space in which the disc will probably be.

The second feature defining the disc area is the determination of a probabilistic model of the disc and surrounding tissues. The disc location itself has the normal probability distribution [9]. An analysis of the nature of bone tissue parts around the disc has found that the location of these tissues has approximately also the normal probability distribution. Based on these characteristics the threshold for segmentation can be determined. The probability distributions of the disc and the surrounding tissues in a limited circular area can be expressed by the image histogram which is shown in Fig.6.

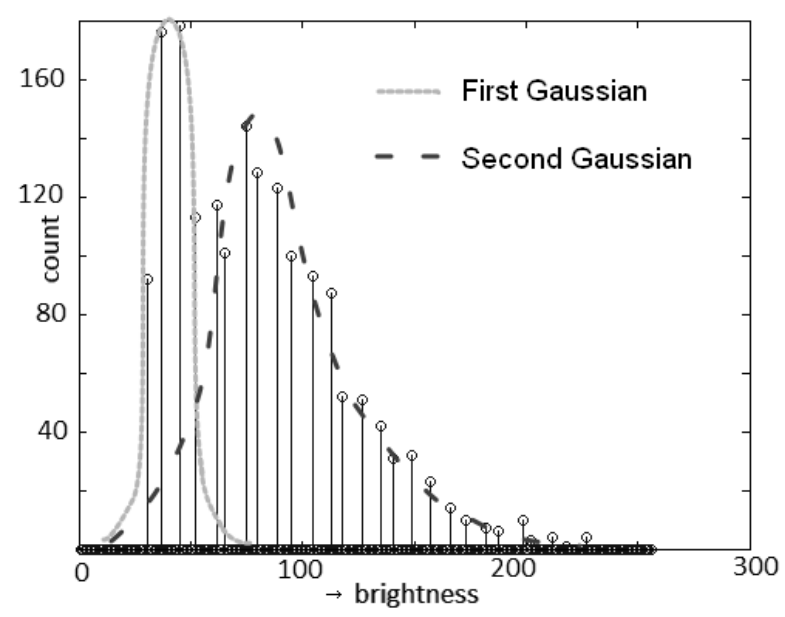

Fig.6. The histogram of the disc image and surrounding tissues limited in an TMJ disc space.

To find the probability function of each tissue from the image histogram, a genetic algorithm can be used. The probability of the occurrence of each part has the normal probability distribution (normalized Gaussian distribution), and the probability density is defined as [16]:

$$
f(x)=\frac{1}{\sigma \sqrt{2 \pi}} \mathrm{e}^{\frac{(x-\mu)^{2}}{2 \sigma^{2}}},
$$


where $\sigma$ is the standard deviation, and $\mu$ is the average. A mixture of Gaussian functions can be defined as:

$$
p_{k}(x)=\sum_{k=1}^{K} \omega_{k} f_{k}(x)
$$

where $\omega_{k}$ denotes the frequency of occurrence.

A matrix, which is set up where individual columns will contain the parameters $\mu, \omega, \sigma$ of the individual Gaussian functions, can be used as the genome. In our case, we have two Gaussian functions [18]. One of them expresses the disc and the second the surrounding tissues. The quality of the genome is determined as an error of the least square (Least Square Error-LSE) approximation of the histogram and can be defined as:

$$
e_{\mathrm{LSE}}=\sum_{i=1}^{n}\left(\tilde{y}_{i}-y_{i}\right)^{2}
$$

where $\tilde{y}_{i}$ is the estimated value for each point of the histogram [6], [10], $y_{i}$ is the value of the original function of the histogram obtained from image brightness, and $n$ denotes the number of brightness components that determine the length of the histogram. Therefore, we compare two functions where one is given by the image histogram and the other is set using the genetic algorithm, while error $e_{\mathrm{LSE}}$ compares how much the functions resemble each other. With increasing number of generations, the generated function will increasingly resemble the function obtained from the image. This is achieved by hybridizing the genomes in descending order after each calculation step, with the following distance functions being used [15]:

$$
\begin{aligned}
& N_{i}+1=\lambda N_{i}(\text { more appropriate })+ \\
& (1-\lambda) N_{i}(\text { less suitable })
\end{aligned}
$$

where $\lambda$ is a decimal number indicating the fitness function of individuals $N_{i}$. A new individual is tested using error $e_{\mathrm{LSE}}$ and is compared with its parents. If one of the individuals is better than its parent, it replaces the parents for the next step. Otherwise, the individual is mutated. If $\mu$, for example, reaches the correct value, this parameter remains unchanged. The parameters $\sigma$ and $\omega$ are then being changed for as long as possible, until they are the closest to the value obtained from the image. The mutated individuals are re-tested by $e_{\mathrm{LSE}}$, and if their properties are not improved, they are stored as unmodified individuals. In the end, after a certain number of generations, the modelled function exhibits enough similarities with the measured function. The algorithm converges to optimum solution within a few hundred generations and reaches a minimum error of similarity between the functions of less than $1 \%$, [16].

When the thresholding operation has been performed, the operation of expansion is followed by an operation of conclusion. The binary image obtained is tested for spatial continuity with the help of Eq.1. Gradually, the area in which the disc is most likely to be found is thus delineated.
After several iterations, spatial coherence combined with thresholding is examined. An example of the results obtained is shown in Fig.7.

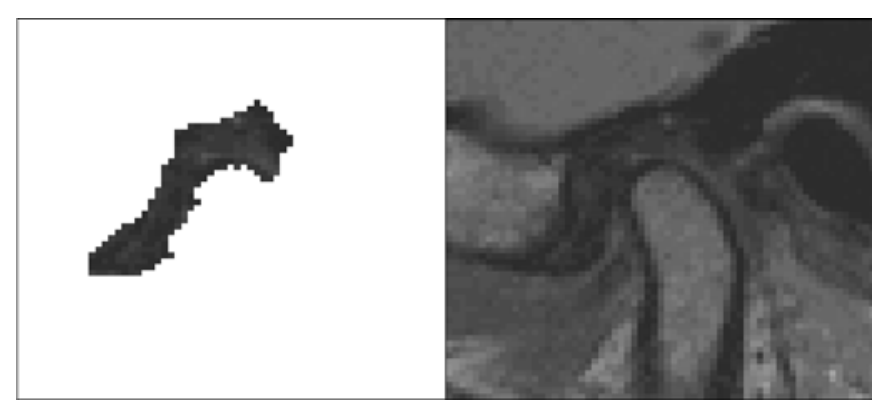

Fig.7. An example of the segmentation by reducing the disc area.

\section{COMPARISON OF PROPOSED METHOD WITH SEGMENTATION METHODS CURRENTLY USED}

These days, several methods are used for the segmentation of biomedical images. The most commonly used methods are the marker-controlled watershed method, the $k$-mean clustering method, and recently also the level-set-function method. We will compare the most frequently used methods with our newly proposed method.

The marker-controlled watershed method was chosen as the first method. The image is treated as a surface, where the bright areas are "mountains" and the dark areas represent "valleys". Local extremes are searched for using an algorithm, and so-called "dams" are formed. Water then starts flowing into the designed places and these areas are flooded. The flooded areas then define the image segments. This method is not very effective for images that contain noise, and it is used a lot for the segmentation of MR brain images. Unfortunately, the method is very sensitive to noise, and noise occurs in the tissue surrounding the TMJ disc. Therefore, it is difficult to determine the boundaries of the end of flooding. The results of the method used are shown in Fig.8.
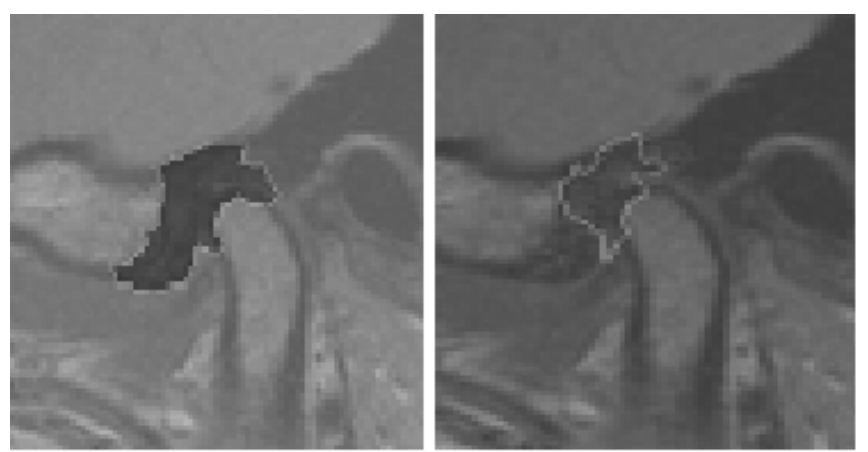

Fig.8. An example of noise influence on the marker-controlled watershed method (the left image has no noise while the right image contains noise).

The second segmentation method tested, which is often used for biomedical images, is the $k$-mean clustering method. The method is based on the principle of determining the centre of a group of pixels with similar 
characteristics. In our case, the picture brightness is the selected feature. One of the important parameters of the method is the number of groups into which the pixels are separated (it is the number of colours, into which the image is divided). We search for the centre of these groups. If we use this method for the segmentation of the TMJ disc, it can be shown that a different number of groups needs to be set for each slice. Setting the number of groups must be carried out intuitively, and it is difficult to automate it. Pictures in Fig.9 show the results of the $k$-mean clustering method. We can see that the same parameters yield different results for different layers.

As can be seen from Fig.9, there is, in addition to the different parameters in individual layers, also the problem of discontinuity in the area found. This means that the region boundaries are not precisely defined and extend to parts that do not belong to the TMJ disc. This serious problem is solved by the third method of segmentation tested, i.e., the level-set-function method. The method is based on the numerical solution of discretized partial differential equations. Steady-state solution gives a curve that encloses the object on the basis of image information.
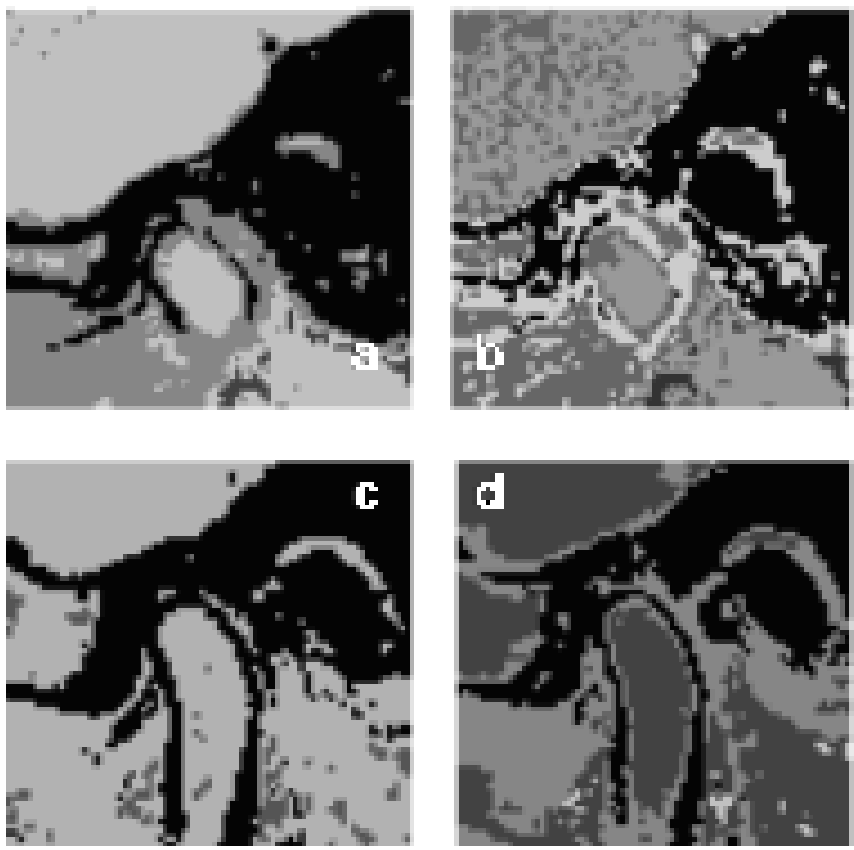

Fig.9. Two different slices segmented by $k$-mean clustering method with different numbers of colours (Fig.a-4 colours, Fig.b-5 colours, Fig.c-3 colours, and Fig.d-4 colours).

Recently, all these methods have been increasingly used, especially for the segmentation of medical images. They are based on the so-called level-set equation [11]:

$$
\frac{\partial \phi}{\partial t}+F|\nabla \phi|=0
$$

where $\phi(t, x, y)$ is the level-set function, which evolves in time $t$ according to speed function $F$. A cut of the multidimensional level-set function at zero level yields a curve which is expressed by the relation:

$$
C(t)=\{(x, y) \mid \phi(t, x, y)=0\}
$$

This method exhibits good properties also in the case of TMJ disc segmentation but is sensitive to the set parameters of the level-set function, especially to the set convergence boundary. The level-set algorithm used for testing the disc segmentation is a state-of-the-art algorithm described in Chapter 8 in [28]. This implementation is based on the following PDE update:

$$
\Phi(i)=\Delta \operatorname{Tg}(i)\left(W_{a} F_{a}|\nabla \Phi|+W_{c} F_{c}|\nabla \Phi|\right)
$$

with

$$
g(I)=\frac{1}{1+2\left(\nabla I^{*}+g\right)}
$$

where $\nabla I^{*}$ is the difference of smoothed (Gaussian blurred) image, and the value of $\mathrm{g}$ is derived from

$$
g=\left\{\begin{array}{l}
0-\text { if grey value }<\text { preset grey value. } \\
\text { grey value }
\end{array}\right.
$$

and

$$
\Delta T=\frac{1}{6 W_{a} W_{c}},
$$

where $\Phi(i)$ is Iso-surface at current iteration $i, W_{a}$ is the advection weight, $F_{a}$ is the advection force, $W_{c}$ is the curvature weight, and $F_{c}$ the curvature force. The parameter values used in the segmentation are given in Table 2.

Table 3. Parameters of level-set algorithm

\begin{tabular}{|l|c|}
\hline \multicolumn{1}{|c|}{ Parameter } & Value \\
\hline Advection & 2.20 \\
\hline Propagation & 1 \\
\hline Curvature & 1 \\
\hline Greyscale tolerance & 30 \\
\hline Convergence criterion & 0.05 \\
\hline
\end{tabular}

Fig.10 shows the results of segmentation using the levelset function. It is setting the parameters of this method that can cause great problems, and only partial segmentations of the TMJ disc may occur. A comparison shows that the new method described in Section 3 works with spatial information of each tissue slice and we can achieve excellent results in a relatively short time. Table 3 gives the measured times necessary for processing the individual algorithms. Since the scanning of such tissues as the TMJ disc depends greatly on how the NMR equipment is set, it is important to find methods that are optimized for setting the NMR equipment [13-15]. 


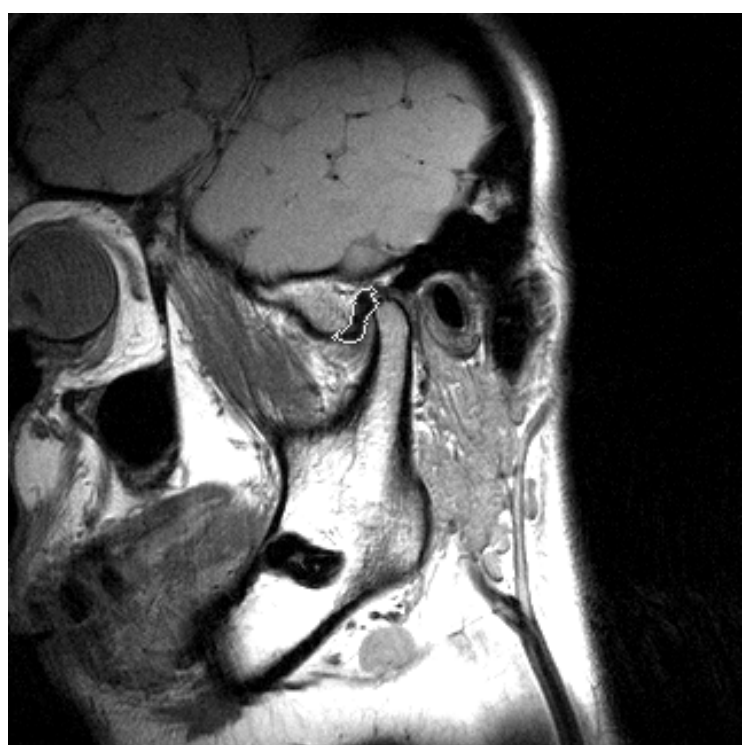

Fig.10. Results of segmentation of disc area, using the level-set function

Table 4. Comparison of the time consumed by individual algorithms

\begin{tabular}{|l|c|c|c|c|}
\hline Algorithm & $\begin{array}{c}\text { Our } \\
\text { algorithm }\end{array}$ & Watershed & $k$-mean & $\begin{array}{c}\text { Level- } \\
\text { Set }\end{array}$ \\
\hline $\begin{array}{l}\text { Time } \\
\text { (ms) }\end{array}$ & 1153 & 468 & 652 & 2530 \\
\hline
\end{tabular}

\section{3D MODELS OF THE TMJ DISC}

\section{A. Extraction of polygonal models}

The most commonly used method for the extraction of polygonal models from medical images is the method of marching cubes. The method is based on the principle of a three-dimensional grid, where cubes move to model the respective surface. Triangles are generated on the basis of a function that determines the extreme points of cubes and these triangles give the best approximation of Iso-surfaces. Iso-surfaces are mathematically defined surfaces determined by normal vectors. Thanks to that, the generation of triangles is always limited to a single cube and the vertices of the triangles generated are always placed in the same cube. As shown in Fig.11, there are 15 potential distributions of the internal and external points of the boundary cube if, in addition, the operation of rotation about the axes and the inverting operation are used. Then the resulting number will rise to 256, [18]. The marching cubes method [20] is very simple and, moreover, very robust. Its disadvantage is that it is very dependent on the chosen grid size. If the basic cube of the grid is small, the accuracy of function approximation increases but the demand on computing operations (memory, power, etc.) will also increase. If the grid size is chosen large, the approximation accuracy of the resulting object will be very low, and vice versa. It is essentially a three-dimensional quantization. If a small quantization step is chosen, then larger storage capacity is necessary and, in addition, the inaccuracies can be rendered due to local noise, [18]. On the other hand, if the quantization step is high, several points can be defined erroneously, and this brings considerable inaccuracy into the 3D model.
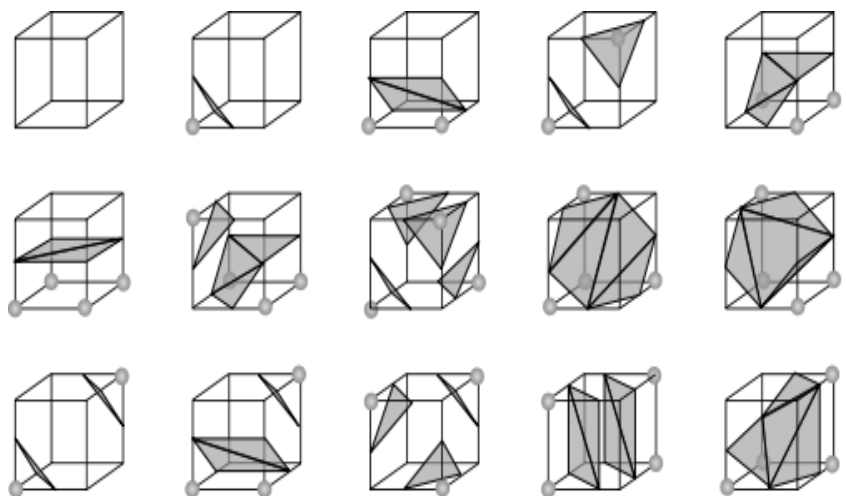

Fig.11. The basic configuration of cubes for the internal and external distribution points, [20].

Fig. 12 shows the difference between the 3D model for low image resolution (measured without microscopic coil) and the 3D model for higher image resolution (measured with microscopic coil). In the 3D model measured without microscopic coil a sharp edge can be seen, which appears due to the small number of slices, [21].
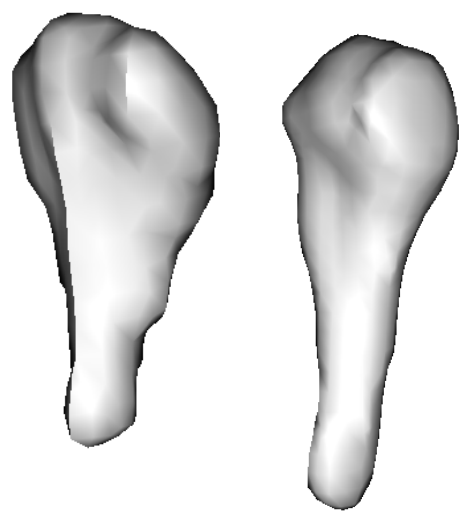

Fig.12. A comparison of the 3D models for both types of image. On the left you can see the 3D model obtained from measurement without microscopic coil. On the right you can see the 3D model obtained from measurement with microscopic coil.

\section{B. Smoothing Methods}

A designed polygonal model is represented by an unstructured triangular net, which is defined by vertices, edges and walls [22], [25]. This model is piecewise linear and discontinuous, and therefore it is necessary to use a smoothing algorithm to smooth it. According to reference [27] a general polygonal model can accurately be described mathematically by $n$-tuple $(\mathbf{K}, \mathbf{p})$, where $\mathbf{K} \subseteq 2^{\mathrm{V}}$ is a simplicial complex, $\mathbf{p}: \mathbf{V} \rightarrow \mathfrak{R}^{3}$ is a mapping function of vertex in space, and $\mathbf{V}$ is non-empty set of vertices. A set of edges can then be defined as:

$$
E=\{e \in K \||e|=2\}
$$


and a set of walls can be defined as:

$$
F=\{f \in K|| f \mid=3\}
$$

The simplest algorithm for smoothing is the Laplace algorithm [26]. This algorithm is based on the discrete approximation of the Laplace operator, which is expressed by the formula:

$$
\Delta x_{i}=\sum_{j \in \operatorname{adj}(i)} \omega_{i j}\left(x_{j}-x_{i}\right)
$$

where $x_{j}$ determines the peak area of $a d j\left(x_{i}\right), x_{i}$ determines the peak being edited, and $\omega_{i}$ is the evaluation of the edge located between $x_{i}$ and $x_{j}$. We will follow the convention that $o_{i}$ is the original point, $q_{i}$ is the point before applying the algorithm, and $p_{i}$ is the point after applying the algorithm. Furthermore, the parameter $\lambda$, which denotes a scale factor, is defined on the interval (0.1>. Using these parameters, the algorithm can be described by this equation:

$$
p_{i}=q_{i}+\lambda \cdot \Delta q_{i}
$$

The algorithm described in reference [27] has two significant disadvantages. First, the resultant model volume is smaller, second there are deformed sharp edges which differ from sharp edges produced by noise. For these reasons, an improved algorithm of the type of Laplace-HC method is used. This algorithm modifies the new point position to be closer to the original location, and thus corrects the final volume. The correction is given by the equation:

$$
d_{i}=\beta b_{i}+\frac{1-\beta}{|\operatorname{adj}(i)|} \sum_{j \in \operatorname{adj}(i)} b_{j},
$$

where $b_{i}$ and $b_{j}$ are the correction vectors, and $\beta$ is the correction factor. The Laplace-HC algorithm can set the model volume up to about $97 \%$ of the original volume, as can be seen from the measurement results in [27]. The resulting 3D model before and after the application of the Laplace-HC algorithm is shown in Fig.13. A wire model after the algorithm application is also shown here.
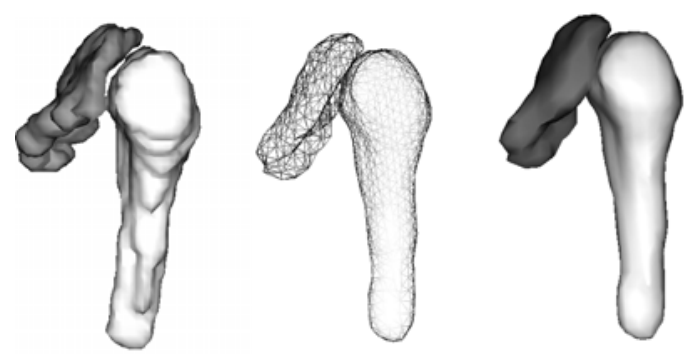

Fig.13. The reconstructed 3D models of the TMJ disc and condyle obtained by MRI measurements with microscopic coil before and after smoothing by the Laplace-HC algorithm (left-the unsmoothed model, middle-the triangular net model, right-the smoothed model).

\section{CONCLUSION}

The paper deals with creating a 3D model of TMJ, the TMJ disc in particular. In the course of implementation we focused on an algorithm that makes use of the combination of a spatial tissue arrangement that restricts the space being scanned and a genetic-algorithm-controlled thresholding. This combination gave rise to an algorithm that can segment the TMJ disc quite well. But since it is human tissue that is being scanned, it cannot be guaranteed that anatomic arrangement of tissues will be maintained; also, even with the tomograph parameters maintained, various errors may appear in the image. The segmentation must, therefore, be performed by an algorithm, always checked, and possibly modified by a specialist in the field of radiology prior to commencing the creation of the 3D model. The main advantage of the algorithm is that it makes minimum demands on the operator (a medical specialist) that will use the algorithm. Since all the required parameters are set while the algorithm is running (via genetic algorithm or spatial arrangement), the operator need not test the optimum parameter combination as is the case, for example, in the level set method (see Table 3).

The proposed method is designed for the sagittal plane only so that further research will go into another two planes (coronal and transverse planes). Combining all the three planes will make it possible to calculate with much precision the space between individual slices. An increased number of slices will also increase the resolution of the 3D model, which will thus better render the actual tissue. Apart from increasing the number of slices, we will be able to segment more precisely the tissue area that has already been modelled for the sagittal plane because we will be able to obtain more information on brightness from the other two planes.

\section{ACKNOWLEDGMENT}

The paper has been supported within the framework of the research project VG20102014033 of the Ministry of Interior of the Czech Republic and the SIX research project (CZ.1.05/2.1.00/03.0072).

\section{REFERENCES}

[1] Adali, T., Calhoun, V.D. (2007). Complex ICA of brain imaging data. IEEE Signal Processing Magazine, 24 (5), 136-139.

[2] Jan, J. (2006). Medical Image Processing, Reconstruction and Restoration : Concepts and Methods. CRC Press.

[3] Vojtisek, L., Frollo, I., Valkovic, L., Gogola, D., Juras, V. (2011). Phased array receiving coils for low field lungs MRI: Design and optimization. Measurement Science Review, 11 (2), 61-66.

[4] Strbak, O., Kopcansky, P., Frollo, I. (2011). Biogenic magnetite in humans and new magnetic resonance hazard questions. Measurement Science Review, 11 (3), 85-91.

[5] Hlavac, V., Sonka, M. (1992). Computer Vision. Prague, CR: Grada. (in Czech) 
[6] Emshoff, R., Brandlmajer, I., Bosh, R., Gerhard, S., Rudish, A., Bertram, S. (2002). Validation of the clinical diagnostic criteria for temporomandibular disorders for the diagnostic subgroup - disc derangement with reduction. Journal of Oral Rehabilitation, 29 (12), 1139-1145.

[7] Manfredini, D., Tognini, F., Melchiorre, D., Cantini, E., Bosco, M. (2003). The role of ultrasonography in the diagnosis of temporomandibular joint disc displacement and intra-articular effusion. Minerva Stomatologica, 52 (3), 93-100, 100-4.

[8] Riha, K. (2008). Advanced Image Processing Techniques. Electronic Texts. Brno University of Technology. (in Czech)

[9] Xu, Ch., Prince, J. Active contours, deformable models and gradient vector flow. Available from www.iacl.ece.jhu.edu/static/gvf/

[10] Cootes, T.F., Taylor, C.J. (2004). Statistical models of appearance for computer vision. Available from www.face-rec.org/algorithms/AAM/app_models.pdf

[11] Osher, S., Fedkow, R. (2003). Level Set Method and Dynamic Implicit Surfaces. New York: Springer.

[12] Bartusek, K., Gescheidtova, E., Mikulka, J. (2010). Data processing in studying biological tissues, using MR imaging techniques. In Proceedings of the $33^{\text {rd }}$ International Conference on Telecommunications and Signal Processing - TSP 2010. Vienna, Austria, 2010, 171-175.

[13] Mikulka, J., Gescheidtová, E., Bartusek, K. (2009). Segmentation of NMR slices and 3D modeling of temporomandibular joint. In Proceedings of the Progress in Electromagnetic Research Symposium. Cambridge, The Electromagnetic Academy, 2009, 165-169.

[14] Mikulka, J., Gescheidtova, E. (2008). Segmentation of NMR image in temporomandibular joint region. In Proceedings of the $31^{\text {th }}$ International Conference on Telecommunications and Signal Processing - TSP 2008. Parádfürdö, Hungary, 2008, 14-17.

[15] Ghassabeh, Y.A., Forghani, N., Forouzanfar, M., Teshnehlab, M. (2005). MRI fuzzy segmentation of brain tissue using IFCM algorithm with genetic algorithm optimization. In IEEE/ACS International Conference on Computer Systems and Applications AICCSA '07, 13-16 May 2007. IEEE, 665-668.

[16] Moller, R., Zeipelt, R. (2001). Automatic segmentation of 3D-MRI data using a genetic algorithm. In Proceedings of the International Workshop on Medical Imaging and Augmented Reality - MIAR '01, 10-12 June 2001. IEEE, 278-282.
[17] Krsek, P. (2001). Direct creating of FEM models from $C T / M R$ data for biomechanics applications. Edition PhD Thesis, Brno University of Technology. (in Czech)

[18] Schroeder, W.J., Zarge, J.A., Lorensen, W.E. (1992). Decimation of triangle meshes. In SIGGRAPH '92 : Proceedings of the $19^{\text {th }}$ Annual Conference on Computer Graphics and Interactive Techniques. New York: ACM, 65-70.

[19] Garland, M., Heckbert, P. (1997). Surface simplification using quadric error metrics. In SIGGRAPH '97 : Proceedings of the $24^{\text {th }}$ Annual Conference on Computer Graphics and Interactive Techniques. New York: ACM, 209-216.

[20] Lorensen, W.E., Cline, H.E. (1987). Marching cubes: A high resolution $3 \mathrm{D}$ surface construction algorithm. In SIGGRAPH '87 : Proceedings of the $14^{\text {th }}$ Annual Conference on Computer Graphics and Interactive Techniques. New York: ACM, 163-169.

[21] Taubin, G. (1995). A signal processing approach to fair surface design. In SIGGRAPH '95 : Proceedings of the $22^{\text {th }}$ Annual Conference on Computer Graphics and Interactive Techniques. New York: ACM, 351358.

[22] Vollmer, J., Mencel, R., Müller, H. (1999). Improved Laplacian smoothing of noisy surface meshes. Dortmund, Germany: Universität Dortmund. (Research Report No. 711 /1999)

[23] Tomas, X., Pomes, J. (2004). Temporomandibular join. In Mercader, J.M., Viñuela, F. (eds.) Therapeutic and Diagnostic Radiology Department of Barcelona Hospital. Barcelona, Spain: Masson, 403-408. (in Spanish)

[24] Tomas, X., Pomes, J., Berenguer, J., Quinto, L., Nicolau, C., Mercader, J.M., Castro, V. (2006). MR imaging of temporomandibular joint dysfunction: A pictorial review. RadioGraphics, 26 (3), 765-781.

[25] Osher, S., Sethian, J.A. (1988). Fronts propagation with curvature-dependent speed: Algorithms based on Hamilton-Jacobi formulations. Journal of Computational Physics, 79 (1), 12-49.

[26] Pribil, J., Horacek, I., Horak, P. (2011). Two methods of mechanical noise reduction of recorded speech during phonation in an MRI device. Measurement Science Review, 11 (3), 92-98.

[27] Vollmer, J., Mencl, R.A., Müller, H. (1999). Improved Laplacian smoothing of noisy surface meshes. Computer Graphics Forum, 18 (3), 131-138.

[28] Yoo, T.S. (ed.) (2004). Insight into Images : Principles and Practice for Segmentation, Registration and Image Analysis. AK Peters/CRC Press.

Received March 9, 2012. Accepted May 31, 2012. 